\title{
FATORES PROGNÓSTICOS EM PACIENTES SUBMETIDOS À CIRURGIA POR TRAUMA PANCREÁTICO
}

\author{
PROGNOSTIC FACTORS IN PATIENTS UNDERGOING SURGERY FOR \\ PANCREATIC TRAUMA
}

\author{
Luiz Carlos Von Bahten, TCBC-PR ${ }^{1}$; Benjamin Smaniotto, TCBC-PR²; William Kondo, AsCBC-PR ${ }^{3}$; \\ Audrey Tieko Tsunoda, AsCBC-RJ ${ }^{3}$; Thienes Maria da Costa Lima ${ }^{4}$; Mariana Jorge Garcia
}

\begin{abstract}
RESUMO: Objetivo: As lesões traumáticas pancreáticas são pouco freqüentes após trauma abdominal fechado ou penetrante. O objetivo deste estudo retrospectivo é analisar a experiência de um serviço universitário e relatar os fatores prognósticos e o tratamento cirúrgico instituído. Método: Foram selecionados e revisados os prontuários dos pacientes portadores de trauma pancreático durante um período de nove anos em um hospital universitário nível terciário de trauma e os parâmetros analisados foram: mecanismo do trauma, presença de choque na admissão, grau da lesão pancreática, escore de trauma, tratamento cirúrgico, complicações e mortalidade. Resultados: Oitenta e nove pacientes foram identificados e o diagnóstico foi realizado durante a laparotomia em todos os casos. Os traumas abdominais penetrantes foram responsáveis por $67,4 \%$ dos casos. Utilizando a Escala de Lesões de Órgãos, as lesões grau II e III foram as mais comuns e o tratamento foi definido de acordo com o grau e o local da lesão. A mortalidade global foi de $21,3 \%$ e significativamente maior nos pacientes que apresentaram choque na admissão, lesões pancreáticas grau IV e V, e Escore de Gravidade da Lesão (ISS) elevado. Conclusões: A lesão pancreática é um fenômeno raro, porém com elevada mortalidade, que está intimamente relacionada à presença de choque na admissão, ao grau da lesão pancreática e ao escore de trauma (Rev. Col. Bras. Cir. 2004; 31(5): 332-337) - ISSN 0100-6991.
\end{abstract}

Descritores: Pâncreas; Lesão pancreática; Tratamento.

\section{INTRODUÇÃO}

O primeiro relato de trauma pancreático foi descrito por Travers ${ }^{1}$, em 1827, e desde então uma série de artigos vem sendo publicados abordando esse tema. A lesão pancreática é pouco freqüente após traumas abdominais fechados ou penetrantes ${ }^{2-7}$, e tem sido relatada em 0,2 a $12 \%$ dos traumas abdominais fechados graves ${ }^{3-6}$ e em 5 a $7 \%$ dos traumas penetrantes ${ }^{7}$. A maioria das lesões pancreáticas ocorre em homens jovens ${ }^{8}$ e está associada a uma alta incidência de lesões a órgãos adjacentes e estruturas vasculares importantes.

Dentre as várias classificações existentes para graduar a lesão pancreática, a mais comumente utilizada é a da Associação Americana para a Cirurgia do Trauma (American Association for the Surgery of Trauma-AAST) ${ }^{9}$. Esta classificação freqüentemente é usada porque enfoca tanto a localização anatômica e a extensão da lesão, quanto a condição do ducto pancreático. A graduação adequada utilizando as definições da AAST ajuda a determinar o tratamento a ser realizado de acordo com o grau da lesão.

O tratamento do trauma pancreático varia desde estratégias não cirúrgicas até ressecções cirúrgicas amplas ou mesmo controle de danos (damage control), dependendo da gravidade e do local da lesão ${ }^{10}$.
Uma alta morbidade e mortalidade estão associadas ao trauma pancreático ${ }^{11}$, com taxas relatadas de 30 a $50 \%$ e de 10 a $32 \%$, respectivamente ${ }^{2-4,6,11,12}$, tornando imperativo o diagnóstico precoce e o tratamento acurados ${ }^{13-15}$.

Tendo em vista a relativa escassez de artigos evidenciando a conduta no trauma pancreático, relatamos a experiência de nove anos de um centro de trauma nível I, enfatizando os fatores prognósticos e o tratamento cirúrgico de acordo com o mecanismo do trauma e a gravidade da lesão.

\section{MÉTODO}

Foram selecionados e revisados os prontuários dos pacientes portadores de lesões pancreáticas durante um período de nove anos em um hospital universitário nível terciário de trauma e os seguintes parâmetros foram analisados: idade, sexo, mecanismo de trauma (penetrante vs. fechado), presença de choque na admissão, localização anatômica da lesão pancreática, lesões associadas, escores de trauma, tratamento cirúrgico, complicações e mortalidade.

As complicações abdominais foram definidas como a presença de abscesso intra-abdominal, formação de fístula pancreática, pancreatite e infecção de sítio cirúrgico. Fístula

1. Cirurgião Geral do Hospital Universitário Cajuru; Professor Titular da Disciplina de Cirurgia Geral da Pontifícia Universidade Católica (PUCPR) e Professor Adjunto da Disciplina de Cirurgia Geral da Universidade Federal do Paraná (UFPR).

2. Cirurgião Geral e Chefe do Serviço de Cirurgia Geral do Hospital Universitário Cajuru; Professor Adjunto da Disciplina de Cirurgia Geral da Pontifícia Universidade Católica (PUC-PR).

3. Residente de Cirurgia Geral do Hospital Universitário Cajuru e da Irmandade Santa Casa de Misericórdia de Curitiba (Aliança Saúde - PUC-PR).

4. Acadêmica de Medicina da Pontifícia Universidade Católica (PUC-PR) 
Tabela 1 - Escala de graduação do trauma pancreático segundo a American Association for the Surgery of Trauma.

\begin{tabular}{|c|c|}
\hline Grau & Descrição da Lesão \\
\hline \multicolumn{2}{|l|}{ I } \\
\hline Hematoma & Contusão menor sem lesão ductal \\
\hline Laceração & Laceração superficial sem lesão ductal \\
\hline \multicolumn{2}{|l|}{ II } \\
\hline Hematoma & Contusão maior sem lesão ductal ou perda tecidual \\
\hline Laceração & Laceração maior sem lesão ductal ou perda tecidual \\
\hline \multicolumn{2}{|l|}{ III } \\
\hline Laceração & Transecção distal ou lesão parenquimatosa com lesão ductal \\
\hline \multicolumn{2}{|l|}{ IV } \\
\hline Laceração & Transecção proximal (à direita da veia mesentérica superior) ou lesão parenquimatosa envolvendo a ampola \\
\hline \multicolumn{2}{|l|}{$\mathrm{V}$} \\
\hline Laceração & Destruição maciça da cabeça do pâncreas \\
\hline
\end{tabular}

pancreática foi definida como a presença de mais de $50 \mathrm{ml} /$ dia de drenagem de secreção com níveis elevados de amilase (mais do que duas vezes a concentração sérica) durante pelo menos três dias consecutivos ${ }^{7}$. Pancreatite foi definida como o aumento sérico da amilase por mais de três dias associado a achados clínicos ${ }^{7}$. Outras complicações incluíram pneumonia e infecção de trato urinário.

A graduação da lesão pancreática foi determinada de acordo com a Escala de Lesão ao Órgão (Organ Injury Scaling) da American Association for the Surgery of Trauma (AAST) ${ }^{9}$ (Tabela 1).

Os testes exato de Fisher, $\mathrm{t}$ de Student e não paramétrico de Mann-Whitney foram utilizados para as comparações quando apropriados. O valor de $p<0,05$ foi considerado estatisticamente significativo. Os dados estão representados pela média \pm desvio padrão.

\section{RESULTADOS}

Durante o período de estudo, oitenta e nove pacientes foram identificados como portadores de trauma pancreático. Oitenta e um (91\%) eram do sexo masculino e a média de idade foi de 30,9 anos (variando de sete a 84 anos). Vinte e nove pacientes $(32,6 \%)$ apresentaram trauma abdominal fechado e os 60 casos restantes eram portadores de trauma penetrante $(67,4 \%)$, incluindo $34(38,2 \%)$ ferimentos por arma de fogo e $26(29,2 \%)$ ferimentos por arma branca. Em todos os casos o diagnóstico de lesão pancreática foi feito durante a laparotomia exploradora.

\section{Choque na admissão}

Vinte e sete pacientes apresentavam pressão sistólica menor do que $90 \mathrm{mmHg}$ na admissão e foram considerados em choque. Os pacientes admitidos em choque apresentaram um risco signitivamente maior de óbito quando comparado aos pacientes hemodinamicamente estáveis $(p<0,0001)$ (Tabela 2).

\section{Localização e graduação da lesão pancreática}

O corpo e a cauda do pâncreas foram os locais mais comuns de lesão $(n=70)$. A cabeça pancreática foi atingida em apenas 19 casos. As lesões pancreáticas grau III foram as mais freqüentes, como demonstrado na Tabela 3, e a taxa de mortalidade apresentou uma correlação importante com a gravidade da lesão pancreática. A lesão mais freqüente no grupo de pacientes sobreviventes foi a de grau II e naqueles que evoluíram para óbito, grau III $(p<0,01)$.

\section{Escores de trauma}

Revised Trauma Score (RTS), Injury Severity Score (ISS) e Trauma Score - Injury Severity Score (TRISS) foram calculados para todos os pacientes, e correlacionados com a mortalidade.

Amédia do RTS foi de 7,2 $\pm 1,1$ (variação: 2,6-7,8). Os sobreviventes apresentaram valores de RTS superiores aos não sobreviventes $(7,5 \pm 0,7$ vs. $6,1 \pm 1,6 ; p<0,01)$.

A média do ISS foi 20,2 $\pm 10,4$ (variação: $3-57$ ). Os não sobreviventes apresentaram maior ISS do que os sobreviventes $(28,4 \pm 12$ vs. $17,9 \pm 8,7 ; p<0,0001)$.

Tabela 2 - Presença de choque na admissão $(n=27)$.

\begin{tabular}{lccc}
\hline & $\begin{array}{c}\text { Pressão arterial } \\
\text { sistólica } \\
<\mathbf{9 0 m m H g}\end{array}$ & $\begin{array}{c}\text { Pressão arterial } \\
\text { sistólica } \\
\mathbf{2 9 0 m m H g}\end{array}$ & Total \\
\hline Sobreviventes & 15 & 55 & 70 \\
Óbitos & 12 & 7 & 19 \\
Total & 27 & 62 & 89 \\
\hline
\end{tabular}

Tabela 3 - Mortalidade conforme a graduação da lesão pancreática $(n=89)$.

\begin{tabular}{lccc}
\hline & Sobreviventes & Óbitos & Mortalidade \\
\hline Grau I & 12 & 0 & $0 \%$ \\
Grau II & 25 & 4 & $13,8 \%$ \\
Grau III & 25 & 10 & $28,6 \%$ \\
Grau IV & 7 & 4 & $36,4 \%$ \\
Grau V & 1 & 1 & $50 \%$ \\
Total & 89 & 19 & $21,3 \%$ \\
\hline
\end{tabular}


Tabela 4 - Comparação entre mecanismo de trauma e o número de lesões associadas $(n=89)$.

\begin{tabular}{lcc}
\hline & Penetrante & Fechado \\
\hline $0-2$ & $20(33,3 \%)$ & $19(65,5 \%)$ \\
$3-5$ & $31(51,7 \%)$ & $9(31)$ \\
$>5$ & $9(15 \%)$ & $1(3.5 \%)$ \\
Total & $60(100 \%)$ & $29(100 \%)$ \\
\hline
\end{tabular}

A média do TRISS foi de 0,91 $\pm 0,18$ (variação: 0,18$0,99)$. Novamente, os sobreviventes apresentaram maior TRISS do que os não sobreviventes $(0,95 \pm 0,11$ vs. $0,75 \pm 0,28$; $p<0,01)$.

\section{Lesões associadas e tratamento cirúrgico}

Oitenta e quatro 84 pacientes $(94,4 \%)$ apresentaram lesão pancreática associada a outras lesões intra-abdominais, e o número de órgãos lesados variou de um a oito, com uma media de 2,94 órgãos lesados por paciente. Os cinco pacientes portadores de lesão pancreática isolada foram vítimas de trauma abdominal fechado (17,2\% dos traumas fechados). Analisando os traumas penetrante e fechado, e comparando os mecanismos de trauma com o número de lesões associadas, verificou-se um maior número de lesões a estruturas e órgãos adjacentes no trauma pancreático penetrante $(3,48$ vs 1,42 , respectivamente; $p<0,0001$ ) (Tabela 4).

Os pacientes que morreram apresentaram um maior número de lesões intra-abdominais associadas comparado aos sobreviventes $(3,37$ e 2,83 , respectivamente; $p=0,24)$, no entanto esta diferença não foi estatisticamente significativa.

O fígado foi o órgão intra-abdominal mais frequientemente lesado em associação ao pâncreas. Dentre todas as lesões associadas, apenas a lesão gástrica foi mais comum nos traumas abertos com uma diferença estatisticamente significativa $(p=0,000001)$ (Tabela 5).

A Tabela 6 sumariza o tratamento das lesões pancreáticas em nossa casuística. $\mathrm{O}$ tratamento cirúrgico variou dependendo da localização e do grau da lesão pancreática. $\mathrm{O}$ procedimento mais realizado foi a simples sutura pancreática $(53,9 \%)$. Vinte e quatro $(27 \%)$ pacientes foram submetidos a pancrea-tectomia, com preservação esplênica em apenas três casos.

Lesões pancreático-duodenais combinadas foram encontradas em 20 pacientes $(22,5 \%)$, sendo que a maioria das lesões duodenais foi reparada primariamente. Em dois casos foi realizada a exclusão pilórica.

Apenas dois pacientes com lesão pancreática grau $\mathrm{V}$ foram identificados em nossa série. Um deles apresentava lesão associada de veia porta e veia cava inferior, e foi submetido a controle de danos (damage control). Evoluiu no quarto dia de pós-operatório com trombose mesentérica, indo a óbito no sexto dia após a cirurgia. O outro paciente apresentava destruição maciça da cabeça do pâncreas, sem outras lesões associadas. Encontrava-se estável durante a cirurgia e foi submetido à cirurgia de Whipple (duodenopancreatectomia). Evoluiu no pós-operatório com fístula pancreática no dia 7, mas apresentou evolução favorável com fechamento espontâneo da fístula, recebendo alta hospitalar 36 dias após a cirurgia.

Tabela 5 - Órgãos intra-abdominais lesados no trauma pancreático.

\begin{tabular}{lcccccl}
\hline & Penetrante & Fechado & Total & \% dos TP & \% dos TC & Valor dop \\
\hline Fígado & 30 & 10 & 40 & $50 \%$ & $35 \%$ & 0,124 \\
Estômago & 32 & 1 & 33 & $53,3 \%$ & $3,5 \%$ & 0,000001 \\
Intestino grosso & 14 & 7 & 21 & $23,3 \%$ & $24,1 \%$ & 0,56 \\
Duodeno & 15 & 5 & 20 & $25 \%$ & $17,3 \%$ & 0,296 \\
Baço & 10 & 8 & 18 & $17 \%$ & $27,6 \%$ & 0,18 \\
Rim & 14 & 4 & 18 & $23,3 \%$ & $13,8 \%$ & 0,22 \\
Intestino delgado & 13 & 3 & 16 & $21,7 \%$ & $10,4 \%$ & 0,156 \\
Vesícula biliar & 5 & 1 & 6 & $8,4 \%$ & $3,5 \%$ & 0,35 \\
\hline
\end{tabular}

$\mathbf{T P}=$ Traumas penetrantes com lesão do órgão específico.

$\mathbf{T C}=$ Traumas contusos com lesão do órgão específico.

Tabela 6 - Opções terapêuticas conforme o grau da lesão $(n=89)$.

\begin{tabular}{lccccccr}
\hline & I & II & III & IV & V & Total & \% \\
\hline Simples drenagem & 6 & 6 & 1 & 0 & 0 & 13 & 14,6 \\
Sutura pancreática & 6 & 22 & 12 & 8 & 0 & 48 & 53,9 \\
Ressecção pancreática & 0 & 0 & 3 & 0 & 0 & 3 & 3,4 \\
Ressecção pancreática e esplenectomia & 0 & 0 & 18 & 3 & 0 & 21 & 23,6 \\
Exclusão pilórica & 0 & 1 & 1 & 0 & 0 & 2 & 2,3 \\
Cirurgia de Whipple & 0 & 0 & 0 & 0 & 1 & 1 & 1,1 \\
Damage control & 0 & 0 & 0 & 0 & 1 & 1 & 1,1 \\
\hline
\end{tabular}




\section{Complicações}

Complicações relacionadas ao pâncreas foram encontradas em 26 dos 82 pacientes $(31,7 \%)$ que sobreviveram mais do que 48 horas. Os sete pacientes que morreram dentro das primeiras 48 horas após o trauma foram excluídos dessa análise. Complicações ocorreram em 15 dos 55 pacientes com ferimentos penetrantes $(27,3 \%)$ e em 11 dos 26 pacientes com trauma abdominal fechado $(42,3 \%)$. Os pacientes que desenvolveram complicações relacionadas ao pâncreas apresentaram um maior tempo de permanência hospitalar (26,88 vs. 11,4 dias; $p<0,0001)$. As principais complicações relacionadas ao pâncreas foram fístula pancreática $(19,5 \%)$, abscesso intraabdominal $(8,5 \%)$ e pancreatite $(8,5 \%)$. Dois pacientes apresentaram pancreatite e fístula pancreática concomitantes, e outros dois evoluíram com pancreatite e abscesso intracavitário.

Complicações não relacionadas ao pâncreas ocorreram em 18 pacientes (22\%), incluindo pneumonia $(8,5 \%)$, infecção de sítio cirúrgico $(8,5 \%)$, infecção de trato urinário $(2,5 \%)$ e atelectasia $(2,5 \%)$.

\section{Mortalidade}

Dezenove pacientes $(21.3 \%)$ faleceram em nossa casuística, sendo sete deles nas primeiras 48 horas após a admissão em decorrência de choque hipovolêmico. Entre os 12 pacientes que sobreviveram mais de 48 horas, foram notificados cinco casos de sepse de origem pulmonar, quatro casos de sepse abdominal (três casos provenientes de abscesso intra-abdominal e um caso de fístula pancreática), dois casos de coagulopatia e falência de múltiplos órgãos e um caso de trombose mesentérica. O tempo médio de permanência hospitalar dos pacientes que sobreviveram mais de 48 horas $(n=82)$ foi de 16,37 dias (16,6 dias para os sobreviventes x 15 dias para os pacientes que faleceram; $p=0,68$ ).

Apesar do maior número de lesões intra-abdominais associadas no trauma penetrante (Tabela 4), não foi demonstrado diferença estatisticamente significativa no que se refere à mortalidade comparando traumas contusos e penetrantes $(p$ $=0,65)$ (Tabela 7).

\section{DISCUSSÃO}

A localização retroperitoneal do pâncreas não só protege o órgão, mas também minimiza os sinais e sintomas de tais lesões, que são freqüentemente vagos e não específicos. Até mesmo uma transecção ductal pode ser assintomática ou oligossintomática no início do processo ${ }^{6,10}$.

Tabela 7 - Mortalidade no trauma abdominal penetrante $e$ fechado.

\begin{tabular}{lccc}
\hline & Penetrante & Fechado & Total \\
\hline Sobreviventes & 48 & 22 & 70 \\
Óbitos & $(80 \%)$ & $(75,9 \%)$ & \\
& 12 & 7 & 19 \\
Total & $(20 \%)$ & $(24,1 \%)$ & \\
\hline
\end{tabular}

O grau de dor abdominal e de sensibilidade abdominal não podem ser usados para discriminar lesões pancreáticas leves e graves ${ }^{16,17}$. A mensuração da atividade sérica da amilase não tem correlação com lesão pancreática nem no trauma aberto nem no fechado.

Entre os exames de imagem disponíveis, a ultrasonografia (FAST examination - Focused Assessment with Sonography for Trauma) não está indicada como uma ferramenta primária para identificar traumas pancreáticos, uma vez que a localização retroperitoneal e a presença de alças intestinais dilatadas e de ar no interior do estômago e duodeno freqüentemente não permitem a visualização do órgão ${ }^{6,10,18}$. A tomografia computadorizada tem sido referida como o melhor exame para a avaliação do retroperitônio em pacientes hemodinamicamente estáveis ${ }^{19}$, apresentando uma sensibilidade global de $71,4 \%{ }^{17}$. A colangiopancreatografia endoscópica retrógrada tem uma acurácia de quase $100 \%$ para traumas ductais ${ }^{20}$, mas é um procedimento extremamente invasivo e pode ser difícil sua realização em situações de emergência ${ }^{6}$. A colangioressonância por sua vez é uma técnica não invasiva e provavelmente seja a modalidade diagnóstica do futuro para a avaliação de lesões dos ductos pancreáticos principais $^{21}$.

A maioria dos traumas pancreáticos é descoberta durante a laparotomia exploradora, indicada em decorrência de lesões de outros órgãos ou de sangramento abdominal ${ }^{18}$. Em nosso estudo, todas as lesões pancreáticas foram identificadas durante a laparotomia. A lesão ductal é o principal fator determinante de morbi-mortalidade, e este é o motivo pelo qual o reconhecimento dessa lesão não pode ser retarda$\mathrm{do}^{3,4,17,22}$.

Uma média de três a 4,1 lesões intra-abdominais associadas ao trauma pancreático é relatada na literatura ${ }^{23}$, o que condiz com a média de 2,94 lesões encontrada em nossa casuística. Lesões pancreáticas isoladas são relatadas em apenas $30 \%$ dos casos de trauma fechado ${ }^{3,17}$ e em cerca de $2 \%$ dos traumas penetrantes, o que pode ser comparado à ocorrência em 17,2\% e 0\% em nossa série, respectivamente. Esta baixa incidência é devido à localização retroperitoneal e à proximidade de múltiplos órgãos e estruturas vasculares importantes $^{3}$. Os órgãos mais comumente afetados incluem fígado, baço, duodeno, estômago e rins ${ }^{4,17}$. Em nossa casuística, fígado, estômago, intestino grosso e duodeno foram os órgãos mais freqüentemente lesados. O estômago foi o único órgão mais lesado no trauma penetrante com uma diferença estatisticamente significativa ( 32 casos vs. $1 ; p=0,000001)$. Os pacientes com trauma fechado e lesões de outros órgãos associadas apresentam uma mortalidade igual ou superior a 20\%, comparada a 3 a 10\% quando o trauma pancreático ocorre isolado ${ }^{24}$. Comparando os casos de trauma abdominal penetrante e fechado, o primeiro apresentou um maior número de lesões associadas ( 3,48 vs. 1,82 , respectivamente; $p<0,0001)$ mas isto não refletiu em uma maior mortalidade ( $20 \%$ vs $24,1 \%$, respectivamente; $p=0,65$ ). Quando comparamos os pacientes que morreram aos sobreviventes, os primeiros apresentaram um maior número de lesões intra-abdominais associadas $(3,37$ vs. 2,83 , respectivamente; $p=0,24$ ), mas esta diferença não foi estatisticamente significativa. 
Todos os pacientes $(\mathrm{n}=89)$ do estudo foram enquadrados na classificação da AAST. Excetuando-se um paciente, todos aqueles $(n=40)$ que apresentaram contusões e lesões parenquimatosas (lesões grau I e II da AAST) foram submetidos a simples drenagem pancreática associada ou não a hemostasia, ligadura dos vasos com sangramento ativo ou sutura do parênquima, como proposto por Novak et $a l^{25}$. O sistema de drenagem fechada parece ser superior aos drenos abertos no que se refere à redução de complicações sépticas ${ }^{26}$, e este é o método de escolha atualmente. Boffard et $a l^{10}$ afirmam que a sutura de lesões parenquimatosas na tentativa de garantir a hemostasia leva à necrose do tecido pancreático; no entanto percebemos uma boa evolução em quase todos os casos de sutura pancreática em nossa amostra. Um paciente apresentando lesão grau II foi submetido à exclusão pilórica em detrimento da lesão duodenal associada.

As lesões grau III $(\mathrm{n}=35)$ foram tratadas de diversas maneiras. Vinte e um pacientes foram submetidos à ressecção pancreática distal associada à drenagem pancreática, sendo que a preservação esplênica foi possível em apenas três casos. Doze pacientes foram tratados através de sutura da lesão e drenagem do pâncreas, um paciente foi manejado com simples drenagem pancreática e um outro foi submetido à exclusão pilórica devido a trauma duodenal severo associado. Quando comparada à simples drenagem, a pancreatectomia distal mostrou uma diminuição no número de complicações pósoperatórias em pacientes hemodinamicamente estáveis ${ }^{2,7,24,27}$. Para os pacientes com múltiplas lesões associadas à tríade hipotermia, acidose e coagulopatia, a drenagem pancreática com controle de danos (damage control) é a melhor escolha no primeiro tempo cirúrgico ${ }^{26}$.

As lesões grau IV ( $\mathrm{n}=11)$ foram manejadas com sutura pancreática e drenagem $(\mathrm{n}=8)$ ou pancreatectomia distal $(n=3)$. As recomendações para essas lesões variam desde ressecções distais ${ }^{23}$ até preservação do segmento distal com drenagem em Y-de-Roux ${ }^{24}$, dependendo da localização precisa da lesão. A preservação de quantidade suficiente de tecido pancreático é importante para manter a função do órgão9. Alguns autores realizam a drenagem da maioria das lesões proximais, mesmo na presença de lesão ao ducto pancreático principal $^{5}$.

As lesões grau V são pouco freqüentes e geralmente requerem duodenopancreatectomia ou diverticulização duodenal, e são acompanhadas por taxas elevadas de mortalidade, uma vez que a localização da lesão resulta em uma grande associação com lesões de grandes vasos ${ }^{28,29}$.

Geralmente as complicações pós-operatórias aumentam proporcionalmente à gravidade da lesã ${ }^{26}$. A fístula pancreática foi a complicação pós-operatória mais freqüente $(19,5 \%)$ nos pacientes que sobreviveram mais de 48 horas e é relatada na literatura em 5 a $30 \%$ dos casos de trauma pancreático ${ }^{27,29}$. A maioria das fístulas fecha espontaneamente com drenagem adequada ${ }^{23}$. Os abscessos intra-abdominais se desenvolveram em sete pacientes $(8,5 \%)$ em nossa casuística e comumente ocorrem em até $25 \%$ das lesões pancreáticas. O tratamento para esses pacientes consiste em drenagem percutânea ou cirúrgi$\mathrm{ca}^{26}$. A incidência de pancreatite após trauma pancreática é de cerca de $7 \%{ }^{3}$ e este valor é consistente com nossos achados (sete casos; 8,5\%). Outras complicações (18 pacientes, totalizando $21,9 \%$ ) incluíram sete pneumonias, sete infecções de sítio cirúrgico, duas infecções de trato urinário e duas atelectasias. A média de permanência hospitalar para os pacientes que apresentaram complicações foi maior do que para os demais pacientes (26,88 vs. 11,4 dias; $p<0,0001$ ).

As taxas de mortalidade após traumas penetrante e fechado variam de 13 a $32 \%{ }^{9}$ e de $10 \%$ a $30 \% \%^{2-5,7,16,17,23}$, respectivamente. Estes números são consistentes com a mortalidade de $20 \%$ e $24,1 \%$, respectivamente, encontrada em nossa série. A mortalidade global nos nossos pacientes foi de 21,3\% (19 pacientes dentre 89).

Observamos que o tratamento das lesões pancreáticas varia desde a simples drenagem até procedimentos bastante complexos, dependendo do local e da gravidade da lesão. A morbi-mortalidade é bastante elevada e os fatores prognósticos mais importantes incluem a presença de choque na admissão, o grau da lesão pancreática e os escores de trauma.

\footnotetext{
ABSTRACT

Background: Traumatic lesions of the pancreas following blunt or penetrating abdominal trauma are infrequent. The aim of this retrospective study on traumatic pancreatic injuries was to assess the experience of an academic center and to report prognostic factors and surgical treatment. Methods: Patients with pancreatic injuries were identified during a 9-year period from the registries of a level I trauma center and medical records were reviewed. Parameters analyzed were mechanism of injury, presence of shock, degree of injury, trauma score, operative management, outcome, morbidity and mortality. Results: Eighty-nine patients sustaining pancreatic injuries were identified and diagnosis was made during laparotomy in all cases. Penetrating abdominal trauma was observed in $67.4 \%$ of the cases. Using the Organ Injury Scale, grade II and III wounds were more common and management was defined according to the degree and site of injury. Mortality was $21.3 \%$ and it was significantly higher in patients presenting shock on admission, pancreatic injury grades IV and V and higher Injury Severity Score (ISS). Conclusion: Pancreatic injury is a rare but deadly phenomenon and mortality rate is related to the presence of shock on admission, degree of pancreatic injury and trauma scores.
} 


\section{REFERÊNCIAS}

1. Travers B - Rupture of the pancreas. Lancet, 1827, 12:384.

2. Wisner DH, Wold RL, Frey CF - Diagnosis and treatment of pancreatic injuries. An analysis management principles. Arch Surg, 1990, 125(9):1109-1113.

3. Jones RC - Management of pancreatic trauma. Am J Surg, 1985, 150(6):698-704.

4. Akhrass R, Yaffe MB, Brandt CP, et al - Pancreatic trauma: a ten-year multi-institutional experience. Am Surg, 1997, 63(7):598-604.

5. Patton JH, Lyden SP, Croce MA, et al. - Pancreatic trauma: A simplified management guideline. J Trauma, 1997, 43(2):234241.

6. Cirillo RL Jr, Koniaris LG - Detecting blunt pancreatic injuries. J Gastrointest Surg, 2002, 6(4):587-598.

7. Cogbill TH, Moore EE, Morris JA Jr, et al. - Distal pancreatectomy for trauma: a multicenter experience. J Trauma, 1991, 31(12):1600-1606.

8. Prasad KR, Lodge JP-ABC of diseases of liver, pancreas, and biliary system. Transplantation of the liver and pancreas. BMJ, 2001, 322(7290):845-847.

9. Moore EE, Cogbill TH, Malangoni MA, et al. - Organ injury scaling, II: Pancreas, duodenum, small bowel, colon, and rectum. J Trauma, 1990, 30(11): 1427-1429.

10. Boffard KD, Brooks AJ - Pancreatic trauma - injuries to the pancreas and pancreatic duct. Eur J Surg, 2000, 166(1):4-12.

11. Sims EH, Mandal AK, Schlater T, et al. - Factors affecting outcome in pancreatic trauma. J Trauma, 1984, 24(2): 125-128.

12. Young PR Jr, Meredith JW, Baker CC, et al. - Pancreatic injuries resulting from penetrating trauma: a multi-institution review. Am Surg, 1998, 64(9):838-844.

13. Lucas CE-Diagnosis and treatment of pancreatic and duodenal injury. Surg Clin North Am, 1977, 57(1):49-65.

14. Cogbill TH, Moore EE, Kashuk JL - Changing trends in the management of pancreatic trauma. Arch Surg, 1982, 117(5):722728.

15. Smego DR, Richardson JD, Flint LM - Determinants of outcome in pancreatic trauma. J Trauma, 1985, 25(8):771-776.

16. Craig MH, Talton DS, Hauser CJ, et al. - Pancreatic injuries from blunt trauma. Am Surg, 1995, 61(2):125-128.

17. Bradley EL, Young PR Jr, Chang MC, et al. - Diagnosis and initial management of blunt pancreatic trauma: guidelines from a multiinstitutional review. Ann Surg, 1998, 227(6):861-869.
18. Brooks A, Shukla A, Beckingham I - Pancreatic trauma. Trauma, 2003, 5(1):1-8.

19. Peitzman AB, Makaroun MS, Slasky BS, et al. - Prospective study of computed tomography in initial management of blunt abdominal trauma. J Trauma, 1986, 26(7):585-592.

20. Coppola V, Vallone G, Verrengia D, et al. - Pancreatic fractures: the role of $\mathrm{CT}$ and the indications for endoscopic retrograde pancreatography. Radiol Med, 1997, 94(4): 335-340.

21. Fulcher AS, Turner MA, Yelon JA, et al. - Magnetic resonance cholangiopancreatography (MRCP) in the assessment of pancreatic duct trauma and its sequelae: preliminary findings. $\mathrm{J}$ Trauma, 2000, 48(6):1001-1007.

22. Vasquez JC, Coimbra R, Hoyt DB, et al. - Management of penetrating pancreatic trauma: an 11-year experience of a level1 trauma center. Injury, 2001, 32(10):753-759.

23. Jurkovich GJ, Carrico CJ - Pancreatic trauma. Surg Clin North Am, 1990, 70(3):575-593.

24. Wilson RH, Moorehead RJ - Current management of trauma to the pancreas. Br J Surg, 1991, 78(10):1196-1202.

25. Nowak MM, Baringer DC, Ponsky JL - Pancreatic injuries. Effectiveness of debridement and drainage for nontransecting injuries. Am Surg, 1986, 52(11):599-602.

26. Fabian TC, Kudsk KA, Croce MA, et al. - Superiority of closed suction drainage for pancreatic trauma. A randomized prospective study.Ann Surg, 1990, 211(6):724-730.

27. Ivatury RR, Nallathambi M, Rao P, et al. - Penetrating pancreatic injuries. Analysis of 103 consectuvrs cases. Am Surg, 1990, 56(2):90-95.

28. Mansour MA, Moore JB, Moore EE, et al. - Conservative management of combined pancreatoduodenal injuries. Am J Surg, 1989, 158(6):531-535.

29. Wynn M, Hill DM, Miller DR, et al. - Management of pancreatic and duodenal trauma. Am J Surg, 1985, 150(3):327-332.

Endereço para correspondência:

Rua Martin Afonso, 2642 / 1092

Champagnat

80730-030 - Curitiba - Pr

E-mail: bahten@ @etpar.com.br 